\title{
Clinical efficacy of icotinib in lung cancer patients with different EGFR mutation status: a meta-analysis
}

\author{
Jian Qu ${ }^{1,{ }^{*}}$, Ya-Nan Wang ${ }^{2,}{ }^{*}$, Ping Xu${ }^{1}$, Da-Xiong Xiang ${ }^{1}$, Rui Yang ${ }^{3}$, Wei Wei ${ }^{4}$, Qiang $\mathbf{Q u}^{3}$ \\ ${ }^{1}$ Department of Pharmacy, the Second Xiangya Hospital, Central South University, Institute of Clinical Pharmacy, Central \\ South University, Changsha 410078, P.R.China \\ ${ }^{2}$ Department of Respiratory, Hospital of Laiwu Iron and Steel Co.Ltd, Laiwu 271100, P.R.China \\ ${ }^{3}$ Department of Pharmacy, Xiangya Hospital, Central South University, Changsha 410078, P.R.China \\ ${ }^{4}$ Department of General Surgery, Xiangya Hospital, Central South University, Changsha 410008, P.R.China \\ *These authors contributed equally to this work
}

Correspondence to: Jian Qu, email: qujianstanley@163.com

Qiang Qu, email: quqiang2015@hotmail.com

Keywords: icotinib, epidermal growth factor receptor, meta-analysis, objective response rate, disease control rate

Received: November 11, $2016 \quad$ Accepted: February 07, $2017 \quad$ Published: February 18, 2017

Copyright: Qu et al. This is an open-access article distributed under the terms of the Creative Commons Attribution License (CC-BY), which permits unrestricted use, distribution, and reproduction in any medium, provided the original author and source are credited.

\section{ABSTRACT}

Icotinib is a novel and the third listed epidermal growth factor receptor-tyrosine kinase inhibitors (EGFR-TKIs), which exerts a good anti-tumor efficacy on non-small cell lung cancer (NSCLC). The efficacy of EGFR-TKIs has been shown to be associated with the EGFR mutation status, especially exon 19 deletion (19Del) and exon 21 L858R mutation. Therefore, a meta-analysis was performed to assess the efficacy of icotinib in NSCLC patients harboring EGFR mutations (19Del or L858R) and wild type (19Del and L858R loci wild type). A total of 24 studies were included for comparing the objective response rate (ORR) in the EGFR wild type and mutant patients treated with icotinib. The ORRs of EGFR mutant patients (19Del or L858R) are better than those of $E G F R$ wild type patients $(O R=7.03(5.09-9.71), P<0.00001)$. The pooling ORs from 21 studies on the disease control rate (DCR) in EGFR mutant patients are better than those of EGFR wild type patients ( $O R=10.54(5.72-19.43), P<0.00001)$. Moreover, the ORRs of EGFR 19Del patients are better than those of EGFR L858R patients after pooling ORs of 12 studies (OR = 2.04(1.12-3.73), $P=0.019)$. However, there was no significant difference on DCRs of EGFR 19Del patients and those of EGFR L858R patients $(O R=2.01(0.94-4.32), P=0.072)$. Our findings indicated that compared with EGFR wild type patients, EGFR mutant patients have better ORRs and DCRs after icotinib treatment; EGFR 19Del patients treated with icotinib have better ORRs than EGFR L858R patients. EGFR mutation status is a useful biomarker for the evaluation of icotinib efficacy in NSCLC patients.

\section{INTRODUCTION}

Lung cancer is the leading cause of mortality around the world and non-small cell lung cancer (NSCLC) is up to $85 \%$ of all types of lung cancer [1]. NSCLC mainly includes squamous cell carcinoma, adenocarcinoma and large cell carcinoma. No obvious clinical manifestations were observed at early stage and more than $40 \%$ of NSCLC are metastatic (Stage IV) disease at diagnosis [2].

With in-depth studies of genesis and cancer related signal pathway, epidermal growth factor receptor (EGFR)- dependent pathway was revealed to play important roles in the development and progression of epithelial cells in NSCLC patients [3]. EGFR tyrosine kinase inhibitors (EGFR-TKIs) play important roles in the treatment of advanced NSCLC because of their superior efficacy over than chemotherapy [4]. EGFR-TKIs such as gefitinib and erlotinib were identified to extend survival and increase quality of life in NSCLC patients [4-6]. Icotinib was a novel and the third listed EGFR-TKIs, which could exert a good anti-tumor efficacy on NSCLC [7],especially in the re-treatment of advanced NSCLC [8]. Up to now, it 
has become one of the standard drugs for the treatment of advanced NSCLC in China [9].

The efficacy of EGFR-TKIs has been shown to be associated with the EGFR mutation status, especially exon 19 and exon 21 that are sensitive to targeted drug therapies [4, 7, 10-15]. Moreover, previous studies has revealed that patients treated with icotinib harboring EGFR exon 19 deletion (19Del) had better survival than those harboring exon 21 point mutation (L858R) [16] or there were no difference among the patients harboring 19Del or L858R mutations [7, 17, 18]. Because of the inconsistent results, relative small sample sizes and lack of high quality studies, their conclusions are limited value. Therefore, we reviewed all the publications about icotinib and conducted a meta-analysis to assess the efficacy of icotinib in NSCLC patients harboring EGFR mutations (19Del or L858R) or wild type for these two mutations

\section{RESULTS}

\section{Study review and selection}

The study selection procedure is shown in Figure 1. We searched from the databases including PubMed, EMBASE, Web of Science, Wanfang and Chinese National Knowledge Infrastructure (CNKI) to 14th Oct. 2016. A total of 136 publications were found after excluding the duplicated studies. Then we excluded 62 irrelevant studies, 5 meta-analysis, 9 case reports and 12 basic studies. Forty-eight studies were included for further review. We further excluded the article that has no $E G F R$ status or no clinical indicators including objective response rate (ORR) and disease control rate (DCR). Twenty-four publications having EGFR mutation and wide type data were selected to qualitative synthesis. Fifteen studies having EGFR 19Del and L858R mutation data were included in qualitative synthesis. Finally, 24 studies having ORR values and 21 studies having DCR values in EGFR mutant and wild type patients were enrolled for meta-analysis. Twelve studies having ORR values and 8 studies having DCR values in EGFR 19Del and L858R patients were enrolled for meta-analysis.

\section{Characteristics of included studies}

Not all studies had all clinical indicators data. After summarized, there were 24 publications have ORR data compared with EGFR mutation and wild type patients. Among the 24 publications, except one publication [19], 23 publications have DCR data. There were 12 publications having ORR values and 8 publications having DCR values in EGFR 19Del and L858R patients (Table 1). The characteristics of first author's name, publishing year, region, Newcastle-Ottawa Scale (NOS) score, study design and the number of patients harboring EGFR mutation status were shown in Table 1.

\section{Quality evaluation of enrolled publications}

The details of the quality evaluation of enrolled publications were shown in Figure 2. There were no randomized controlled trials involved in EGFR status on the efficacy of icotinib in NSCLC patients harboring different EGFR mutation status. All studies were cohort trials and retrospective studies. Therefore the risk of bias was high regarding adequate sequence generation and blinding. But other methodological issues present relatively little risk.

\section{EGFR wild type vs. EGFR mutation}

There were 24 publications enrolled for comparing the ORR in EGFR wild type patients and EGFR mutant patients (19Del or L858R). Total patients enrolled in the meta-analysis were 1300 including 825 EGFR mutant patients and $475 E G F R$ wild type patients. There was no heterogeneity among 24 publications $\left(\mathrm{I}^{2}=5.4 \%\right.$, $P=0.387$ ). Therefore, Mantel-Haenszel fixed effects model was used to calculate the pooled odds ratio of included studies. The results showed that the ORRs of EGFR mutant patients are better than those of $E G F R$ wild type patients $(\mathrm{OR}=7.03$ (5.09-9.71), $P<0.00001)$ (Figure 3A).

Among the 24 publications, one publication [19] has no data for DCRs; DCRs were $100 \%$ in EGFR mutant patients and $E G F R$ wild type patients in two publications $[12,20]$. After excluding these 3 publications, 21 publications having 1127 patients including 750 EGFR mutant patients and 377 EGFR wild type patients were enrolled in meta-analysis for DCRs. Because of heterogeneity $\left(\mathrm{I}^{2}=53.2 \%, P=0.002\right)$, Mantel-Haenszel random effects model was used to analyze. The results showed that the DCRs of EGFR mutant patients are better than those of $E G F R$ wild type patients $(\mathrm{OR}=10.54$ (5.72-19.43), $P<0.00001)$ (Figure 3B). According to subgroup analysis for NOS, there was no heterogeneity in NOS 5 and 6 groups $\left(\mathrm{I}^{2}=0.0 \%, P=0.587 ; \mathrm{I}^{2}=0.0 \%\right.$, $P=0.574$, respectively), but NOS 7 groups has heterogeneity $\left(\mathrm{I}^{2}=62.4 \%, P=0.021\right)$. The DCRs of $E G F R$ mutant patients are better than those of EGFR wild type patients according to subgroups for different NOS $(\mathrm{OR}=5.06(1.37-18.7), P=0.015 ; 14.53(8.47-24.9)$, $P<0.0001 ; 10.37(2.25-42.56), P=0.001$, respectively) (Figure 3C).

\section{EGFR 19Del vs. EGFR L858R}

There were 12 publications enrolled for comparing the ORR in EGFR 19Del and EGFR L858R patients. Total patients enrolled in the meta-analysis on ORRs were 555, including 303 EGFR 19Del patients and 252 L858R patients. Because of heterogeneity $\left(\mathrm{I}^{2}=49.7 \%\right.$, $P=0.025)$, a Mantel-Haenszel random-effects model was used to analyze. The results showed that the ORRs 
Table 1: Characteristics of studies included in meta-analysis

\begin{tabular}{|c|c|c|c|c|c|c|c|c|c|c|c|}
\hline \multirow[b]{2}{*}{ Author } & \multirow[b]{2}{*}{ Year } & \multirow[b]{2}{*}{ Region } & \multirow{2}{*}{$\begin{array}{l}\text { Study } \\
\text { design }\end{array}$} & \multirow{2}{*}{$\begin{array}{l}\text { NOS } \\
\text { Score }\end{array}$} & \multicolumn{3}{|c|}{$E G F R$ mutant and wild type } & \multicolumn{3}{|c|}{ EGFR 19Del and L858R } & \multirow[b]{2}{*}{ References } \\
\hline & & & & & $\begin{array}{c}\mathrm{M} / \mathrm{W} \\
\text { number }\end{array}$ & ORR & DCR & $\begin{array}{c}\text { 19Del/L858 } \\
\text { number }\end{array}$ & ORR & DCR & \\
\hline Zhao Q & 2011 & China & $\mathrm{R}$ & 6 & $5 / 14$ & $\mathrm{Y}$ & $\mathrm{Y}$ & $4 / 1$ & $\mathrm{Y}$ & $\mathrm{N}$ & {$[20]$} \\
\hline Zen Xiao-Mei & 2013 & China & $\mathrm{R}$ & 6 & $15 / 3$ & $\mathrm{Y}$ & $\mathrm{Y}$ & & $\mathrm{N}$ & $\mathrm{N}$ & {$[38]$} \\
\hline Yang Xin-Jie & 2013 & China & $\mathrm{R}$ & 6 & $18 / 2$ & $\mathrm{Y}$ & $\mathrm{Y}$ & $10 / 8$ & $\mathrm{Y}$ & $\mathrm{Y}$ & {$[39]$} \\
\hline Yang S & 2016 & China & $\mathrm{R}$ & 7 & $32 / 55$ & $\mathrm{Y}$ & $\mathrm{N}$ & & $\mathrm{N}$ & $\mathrm{N}$ & {$[31]$} \\
\hline Wei Feng-Lu & 2015 & China & $\mathrm{R}$ & 6 & $15 / 15$ & $\mathrm{Y}$ & $\mathrm{Y}$ & & $\mathrm{N}$ & $\mathrm{N}$ & {$[40]$} \\
\hline Wang Tao & 2016 & China & $\mathrm{R}$ & 7 & $38 / 29$ & $\mathrm{Y}$ & Y & $15 / 16$ & $\mathrm{Y}$ & $\mathrm{N}$ & {$[32]$} \\
\hline Tan Fen-Lai & 2012 & China & $\mathrm{R}$ & 7 & $249 / 101$ & $\mathrm{Y}$ & $\mathrm{Y}$ & $72 / 88$ & $\mathrm{Y}$ & $\mathrm{Y}$ & {$[35]$} \\
\hline Sun Jing & 2014 & China & $\mathrm{R}$ & 7 & $34 / 10$ & $\mathrm{Y}$ & Y & & $\mathrm{N}$ & $\mathrm{N}$ & [41] \\
\hline Song Zheng-Bo & 2013 & China & $\mathrm{R}$ & 6 & $36 / 13$ & $\mathrm{Y}$ & $\mathrm{Y}$ & & $\mathrm{N}$ & $\mathrm{N}$ & {$[42]$} \\
\hline Shao L & 2014 & China & $\mathrm{R}$ & 7 & $12 / 16$ & $\mathrm{Y}$ & $\mathrm{Y}$ & & $\mathrm{N}$ & $\mathrm{N}$ & {$[43]$} \\
\hline Ren G-J & 2011 & China & $\mathrm{R}$ & 5 & $7 / 7$ & Y & Y & $3 / 4$ & $\mathrm{Y}$ & Y & [44] \\
\hline Pang Lin-Rong & 2014 & China & $\mathrm{R}$ & 6 & $33 / 18$ & $\mathrm{Y}$ & $\mathrm{Y}$ & $19 / 13$ & $\mathrm{Y}$ & $\mathrm{Y}$ & [21] \\
\hline Nong Jin-Yin & 2013 & China & $\mathrm{R}$ & 6 & $23 / 9$ & Y & Y & $14 / 9$ & Y & $\mathrm{N}$ & {$[22]$} \\
\hline $\mathrm{Na}$ Qin & 2013 & China & $\mathrm{R}$ & 6 & $35 / 11$ & $\mathrm{Y}$ & $\mathrm{Y}$ & & $\mathrm{N}$ & $\mathrm{N}$ & {$[16]$} \\
\hline Ma Xiang-Leiei & 2014 & China & $\mathrm{R}$ & 7 & $40 / 14$ & $\mathrm{Y}$ & Y & & $\mathrm{N}$ & $\mathrm{N}$ & {$[45]$} \\
\hline Liang Shao-Ping-Ping & 2015 & China & $\mathrm{R}$ & 5 & $10 / 2$ & Y & Y & & $\mathrm{N}$ & $\mathrm{N}$ & {$[46]$} \\
\hline $\mathrm{Li} \mathrm{Xi}$ & 2012 & China & $\mathrm{R}$ & 6 & $23 / 36$ & $\mathrm{Y}$ & $\mathrm{Y}$ & $55 / 40$ & $\mathrm{Y}$ & $\mathrm{Y}$ & {$[34]$} \\
\hline $\mathrm{Li} \mathrm{Xi}$ & 2015 & China & $\mathrm{R}$ & 6 & $99 / 25$ & Y & Y & $13 / 9$ & Y & $\mathrm{Y}$ & {$[18]$} \\
\hline Li Ran & 2013 & China & $\mathrm{R}$ & 5 & $23 / 7$ & Y & $\mathrm{Y}$ & $17 / 6$ & Y & Y & {$[47]$} \\
\hline He Xiao-Tin & 2015 & China & $\mathrm{R}$ & 6 & $33 / 13$ & $\mathrm{Y}$ & Y & & $\mathrm{N}$ & $\mathrm{N}$ & {$[48]$} \\
\hline He Chun-Xiao & 2012 & China & $\mathrm{R}$ & 7 & $1 / 4$ & Y & Y & & $\mathrm{N}$ & $\mathrm{N}$ & [49] \\
\hline Guo Lei & 2016 & China & $\mathrm{R}$ & 7 & $21 / 6$ & Y & Y & & $\mathrm{N}$ & $\mathrm{N}$ & {$[50]$} \\
\hline $\mathrm{Gu} \mathrm{A}$ & 2013 & China & $\mathrm{R}$ & 5 & $4 / 2$ & Y & Y & & $\mathrm{N}$ & $\mathrm{N}$ & [51] \\
\hline Chen Xiao-Feng & 2014 & China & $\mathrm{R}$ & 6 & $19 / 63$ & $\mathrm{Y}$ & $\mathrm{Y}$ & & $\mathrm{N}$ & $\mathrm{N}$ & [33] \\
\hline Zhang Xiao-Xue & 2016 & China & $\mathrm{R}$ & 7 & & $\mathrm{~N}$ & $\mathrm{~N}$ & $60 / 44$ & Y & $\mathrm{N}$ & {$[7]$} \\
\hline Shen Yan-Wei & 2016 & China & $\mathrm{R}$ & 6 & & $\mathrm{~N}$ & $\mathrm{~N}$ & $21 / 14$ & $\mathrm{Y}$ & $\mathrm{Y}$ & [17] \\
\hline
\end{tabular}

Abbreviations: Y, Yes; N, NO; M, Mutation; W, wild type; Del, deletion; R, Retrospective study; NOS, Newcastle-Ottawa Scale; ORR, Objective Response Rate; DCR, disease control rate.

of EGFR 19Del patients are better than those of EGFR L858R patients $(\mathrm{OR}=2.04(1.12-3.73), P=0.019)$ (Figure 4A). After excluded one publication [21] that could influence the overall effective size, there was no heterogeneity $\left(\mathrm{I}^{2}=43.8 \%, P=0.058\right)$ and the ORRs of EGFR 19Del patients are better than those of EGFR L858R patients $(\mathrm{OR}=1.48(1.02-2.13), P=0.037))$ (Figure 4B).

Among the 12 publications, one publication [7] has no data for DCRs; DCRs were 100\% in EGFR 19Del and L858R patients in three publications [12, 20, 22]. After excluded these 4 publications, 8 publications containing 392 patients including 210 EGFR 19Del patients and $182 \mathrm{~L} 858 \mathrm{R}$ patients were enrolled in meta-analysis for DCRs. Because there was no heterogeneity $\left(\mathrm{I}^{2}=0.0 \%\right.$,
$P=0.776$ ), a Mantel-Haenszel fixed-effects model was used to analyze. The results showed that there were no significant differences on DCRs of EGFR 19Del patients and L858R patients $(\mathrm{OR}=2.01(0.94-4.32), P=0.072)$ (Figure 4C).

\section{Publication bias and sensitivity analysis}

Publication bias was examined by funnel plots, Egger's test and Begg's test. For pooling ORs analysis for ORRs in EGFR wild type patients and mutant patients, funnel plots, Egger's test $(P=0.09)$ and Begg's test $(P=0.747)$ showed no publication bias (Figure 5A-5C respectively). For pooling ORs analysis for DCRs in $E G F R$ wild type patients and mutant patients, funnel plots 
and Begg's $(P=0.651)$ test showed no publication bias (Figure 5D and 5F, respectively). However, Egger's test $(P=0.003)$ showed publication bias (Figure 5E).

For pooling ORs analysis for ORRs among EGFR 19Del and L858R patients, funnel plots, Egger's test $(P=0.164)$ and Begg's test $(P=0.451)$ showed no publication bias (Figure 6A-6C, respectively). For pooling ORs analysis for DCRs in the EGFR 19Del and L858R patients, funnel plots, Egger's test $(P=0.376)$ and Begg's test $(P=0.711)$ showed no publication bias (Figure 6D-6F, respectively).

Sensitivity analysis results showed that changing the effect models had no significant effects on the pooled ORs and the final strength of the association between EGFR polymorphisms and clinical efficacy of icotinib in lung cancer patients. Moreover, Figure 7A showed the results of sensitivity analysis regarding DCRs of EGFR mutant patients $v s$. EGFR wild type patients. We found that excluded studies did not influence the overall effective size in DCRs analysis of EGFR mutant patients $v S$. EGFR wild type patients. Figure 7B showed one publication (Pang Lin-Rong, 2014) [21] could influence the overall effective size in ORRs analysis of EGFR 19Del and EGFR L858R patients. After we excluded this publication [21], There was no heterogeneity for pooling ORs analysis for ORRs in EGFR $19 \mathrm{Del}$ and EGFR L858R patients $\left(\mathrm{I}^{2}=43.8 \%\right.$, $P=0.058$ ) (Figure 3B).

\section{DISCUSSION}

We carried out this meta-analysis to evaluate the clinical efficacy of icotinib in lung cancer patients with different EGFR mutation status. Our meta-analysis suggests that compared with EGFR wild type patients, EGFR mutant patients have better ORRs and DCRs after icotinib treatment; compared with EGFR L858R patients, EGFR 19Del patients have better ORRs after icotinib treatment.

It is widely accepted that EGFR plays important roles in tumor cell proliferation, invasion, angiogenesis, metastasis and apoptosis [23]. EGFR-TKIs such as gefitinib or erlotinib inhibit tumor cells by blocking the EGFR signaling via binding to ATP binding site of EGFR to increase survival in NSCLC patients $[8,24,25]$. Several clinical studies have confirmed that patients with EGFR mutation have benefited from EGFR-TKIs treatment [26-28]. There were two major EGFR-activating

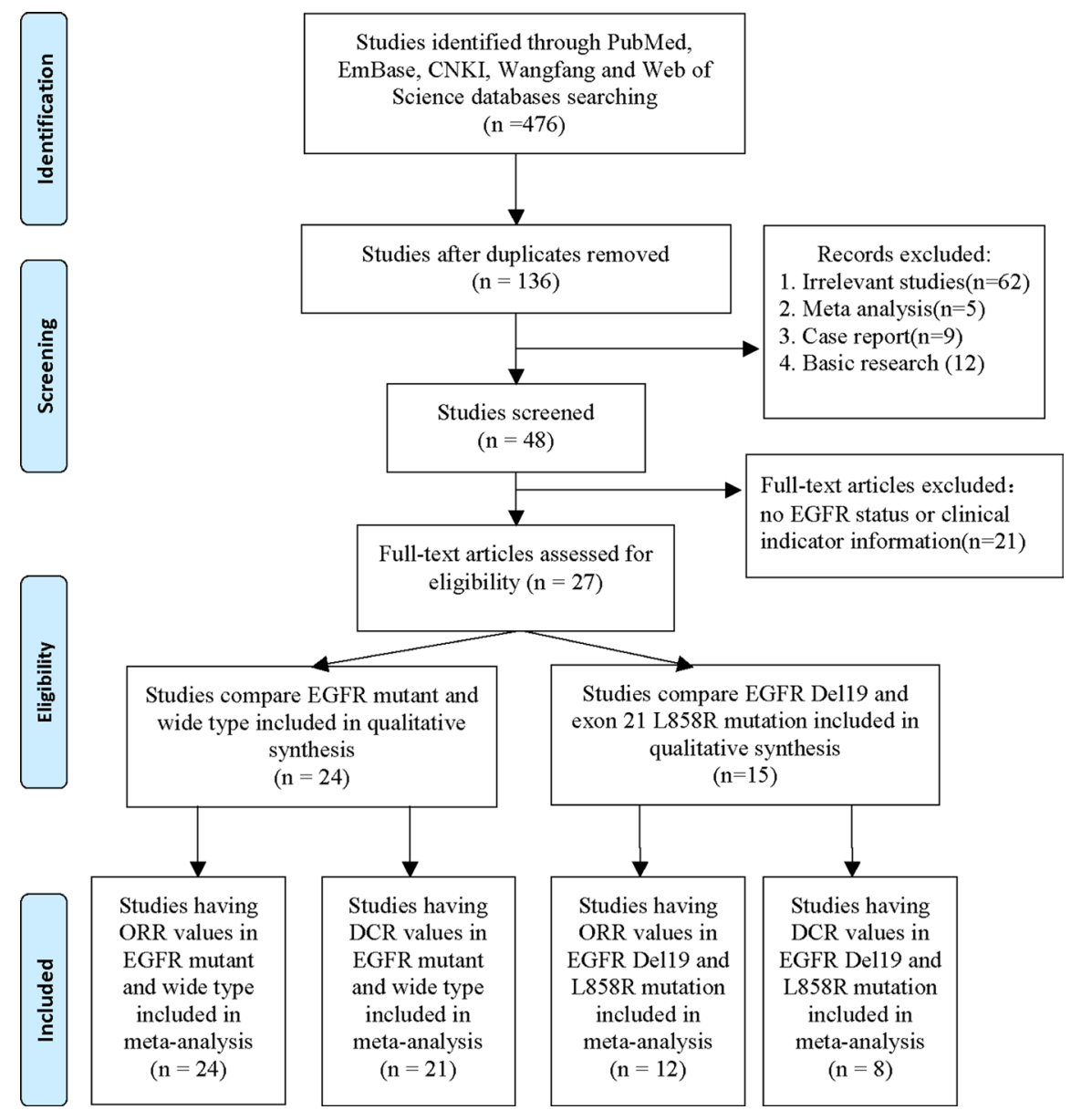

Figure 1: Procedure of article selection. 
Random sequence generation (selection bias)

Allocation concealment (selection bias)

Blinding of participants and personnel (performance bias)

Blinding of outcome assessment (detection bias)

Incomplete outcome data (attrition bias)

Selective reporting (reporting bias)

Other bias

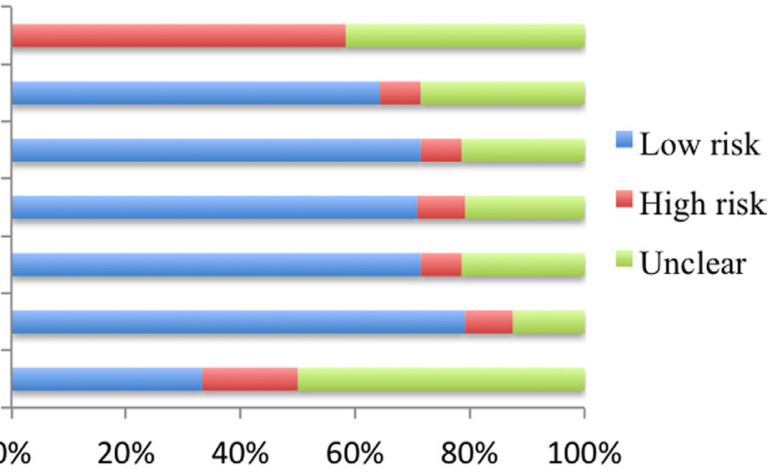

Figure 2: Analysis of risk of bias.

A

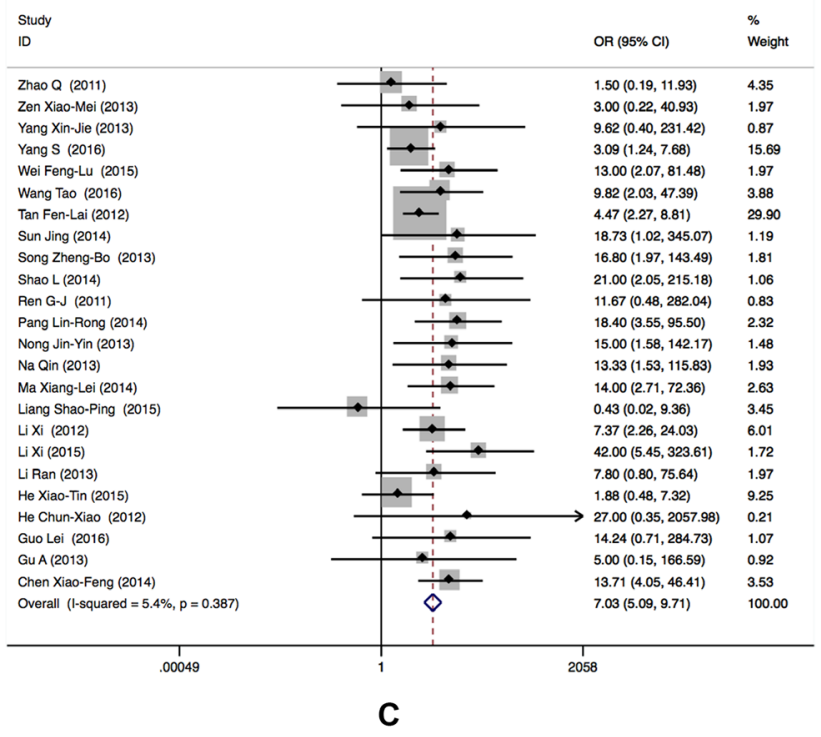

B

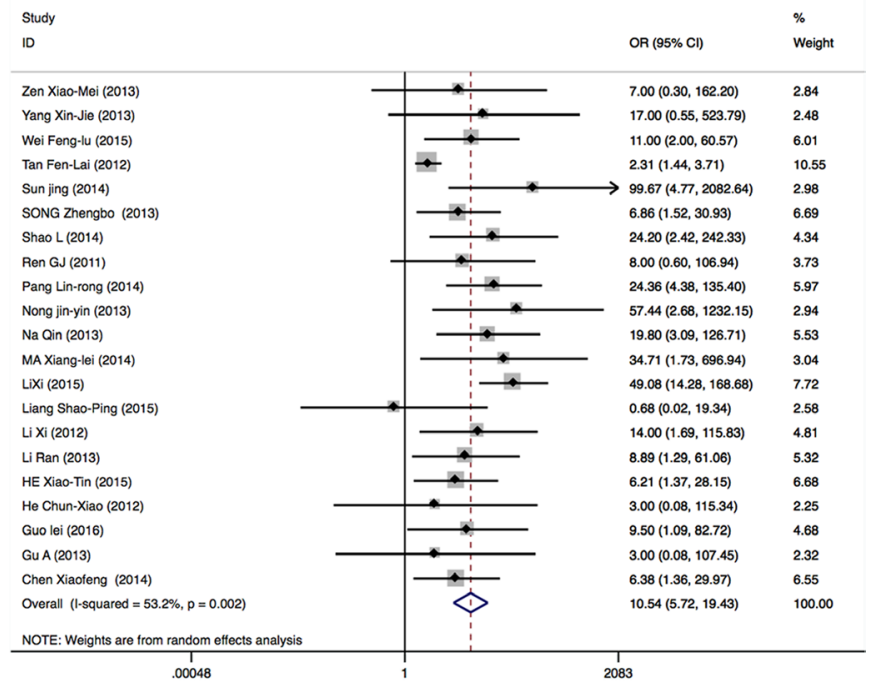

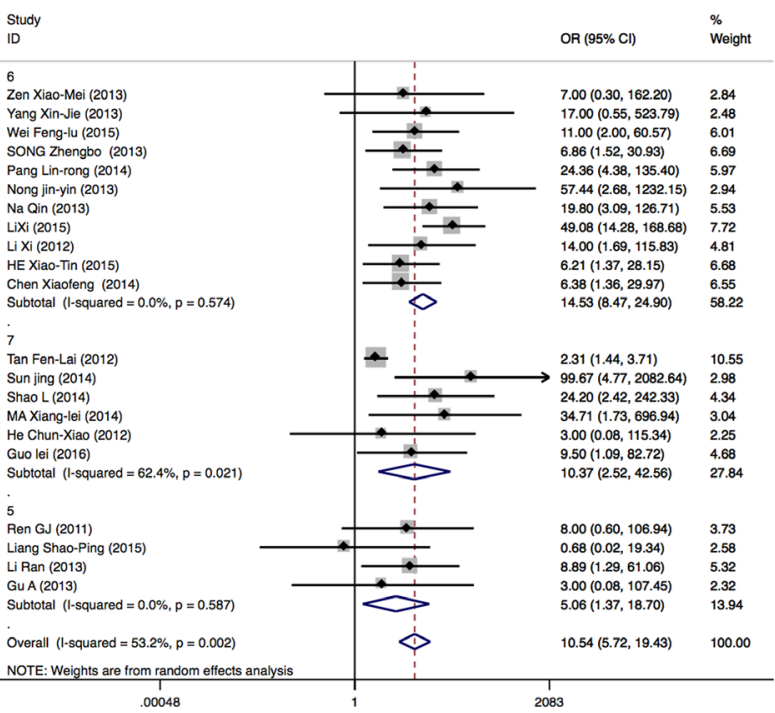

Figure 3: Forest plots of studies evaluating odds ratios of ORRs (A), DCRs (B) and DCRs subgroups analysis according to NOS (C) in EGFR wild type and EGFR mutant patients. OR: odds ratio. 


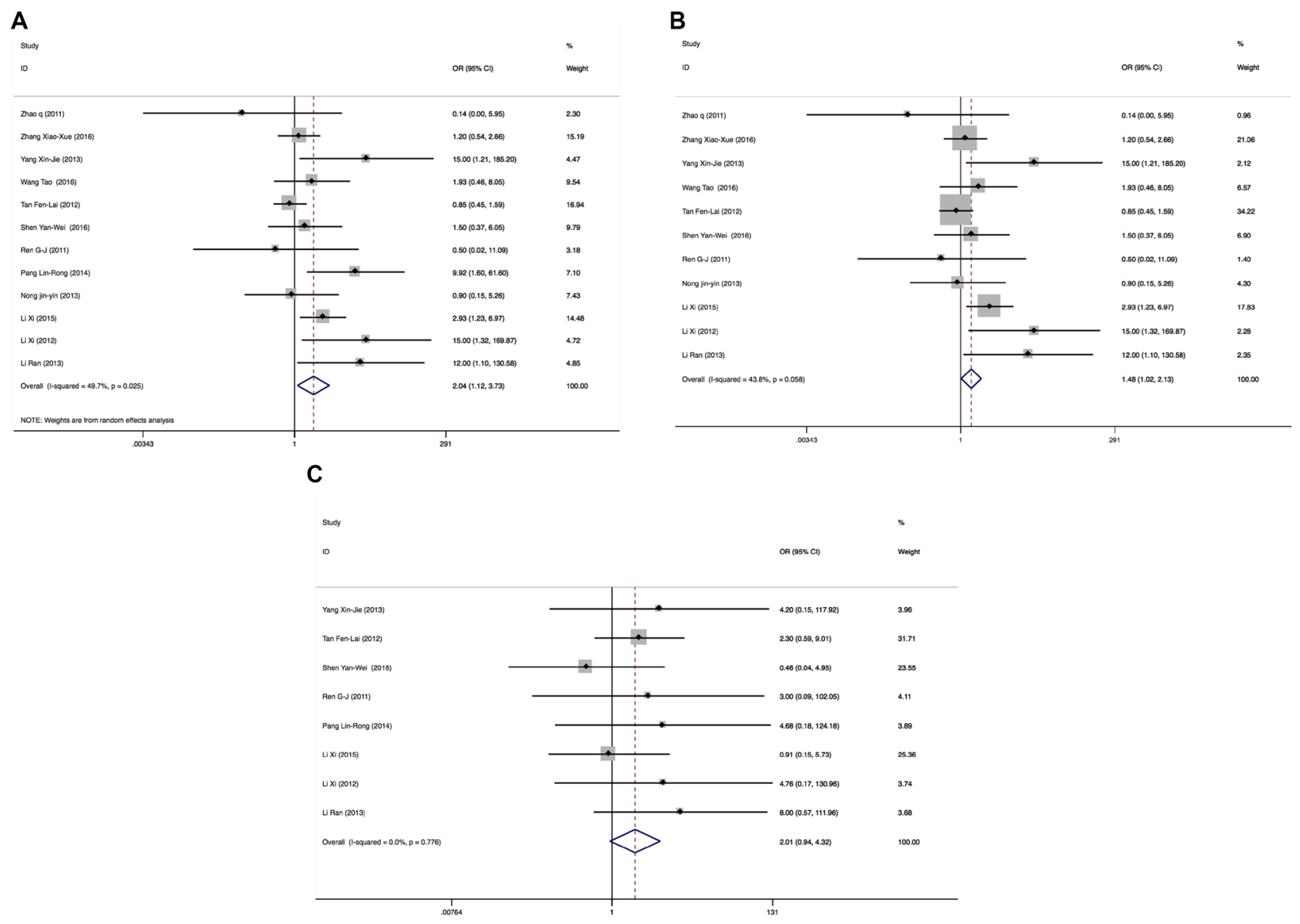

Figure 4: Forest plots of studies evaluating odds ratios of ORRs (A), forest plots of studies excluding one publication that influences the overall effective size evaluating odds ratios of ORRs (B) and DCRs (C) in EGFR 19Del and L858R patients. OR: odds ratio.

A

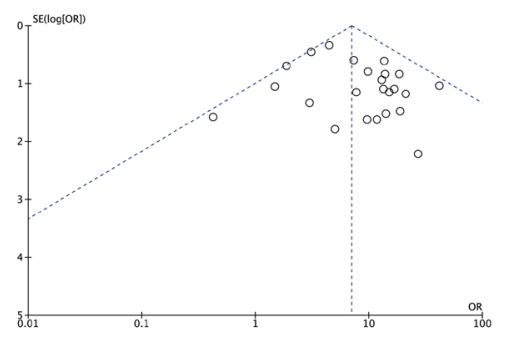

D

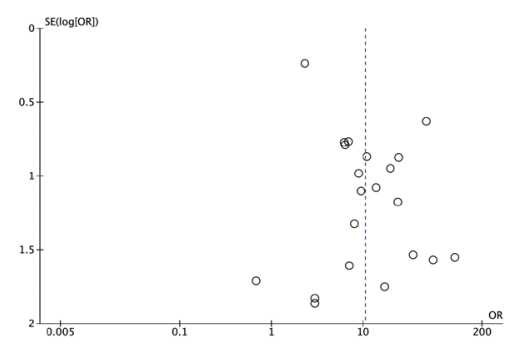

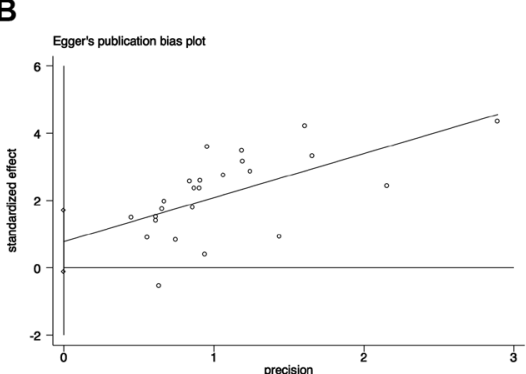

B

$\mathrm{E}$

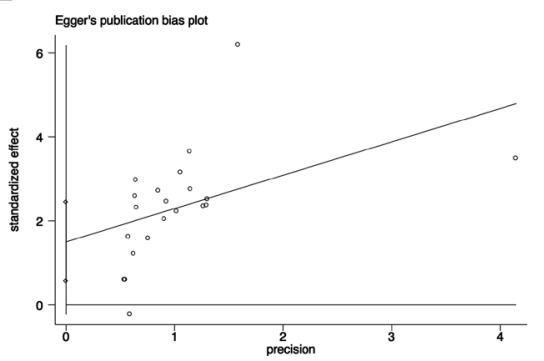

C

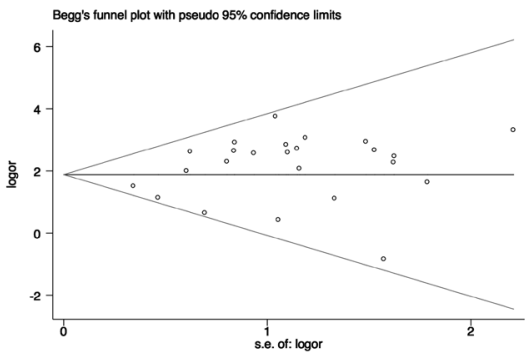

$\mathbf{F}$

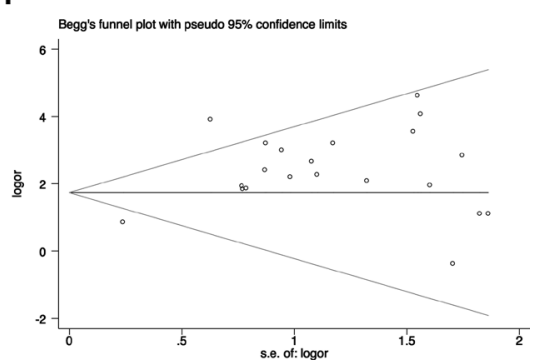

Figure 5: The funnel plots, Egger's test and Begg's test of publication bias in pooling ORs analysis in EGFR wild type and EGFR mutant patients. (A) The funnel plots, (B) Egger's test and (C) Begg's test for pooling ORs of ORRs analysis; (D) The funnel plots, (E) Egger's test and (F) Begg's test for pooling ORs of DCRs analysis. OR: odds ratio; SE: standard error. 
mutations including an in-frame deletion in exon 19 and an L858R substitution in exon 21, which account for about $90 \%$ of all clinically important mutations related to EGFR- TKIs sensitivity [29, 30]. Icotinib is a new type and the third world listed EGFR-TKIs to treat for NSCLC patients [8]. Several studies have revealed that EGFR mutant patients could get more benefit from icotinib treatment than EGFR wild type patients [18, 22, 31-33]. More evidences have the trend to imply that EGFR 19Del patients have better efficacy of icotinib than L858R patients [20, 34, 35]. However, there were some negative results against these views [7, 14, 17]. Furthermore, these studies were small sample size and retrospective study. Whether EGFR mutation status, especial EGFR 19Del and L858R, influence the efficacy of icotinib in NSCLC patients, it is still unclear now. Therefore, we carried out this meta-analysis to figure them out.

Icotinib is a new EGFR-TKI used in the treatment of NSCLC patients and it is only available in China. There were no randomized controlled trials on the efficacy of icotinib in NSCLC patients harboring different EGFR mutation status. Most of studies were cohort and retrospective studies enrolled small sample sizes. Therefore, there were some risks of bias such as high regarding adequate sequence generation and blinding.

The heterogeneities of studies are common in metaanalysis because of the different type of studies, methods or the selected case and controls. In our study, there was no

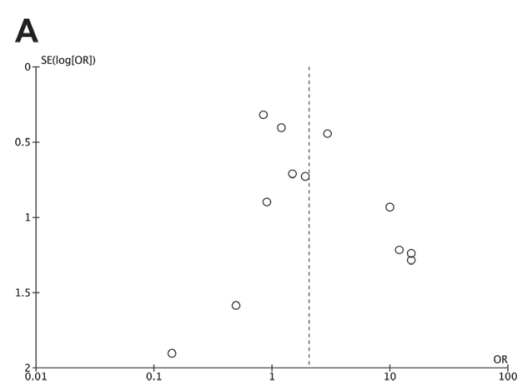

D

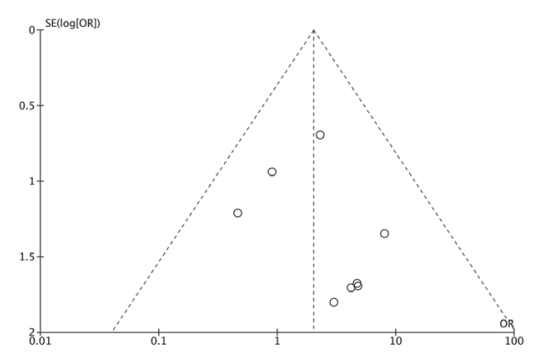

B

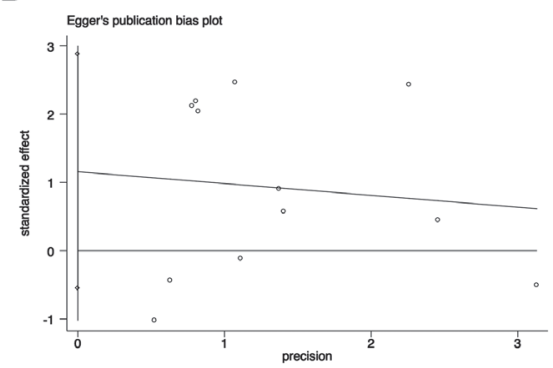

E

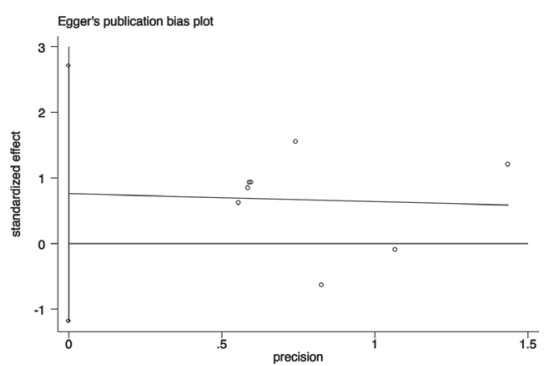

C

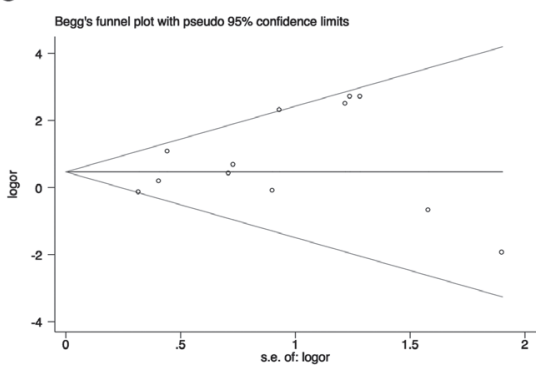

$\mathbf{F}$

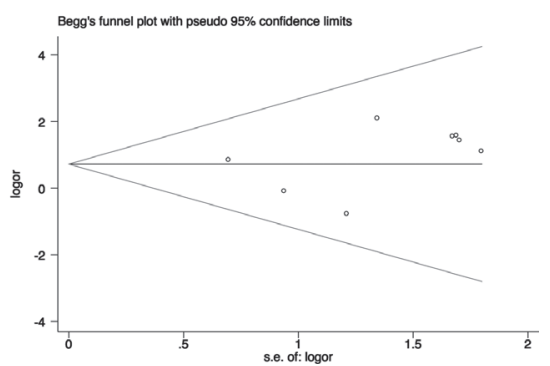

Figure 6: The funnel plots, Egger's test and Begg's test of publication bias in pooling ORs analysis in EGFR 19Del and L858R patients. (A) The funnel plots, (B) Egger's test and (C) Begg's test for pooling ORs of ORRs analysis; (D) The funnel plots, (E) Egger's test and (F) Begg's test for pooling ORs of DCRs analysis. OR: odds ratio; SE: standard error.
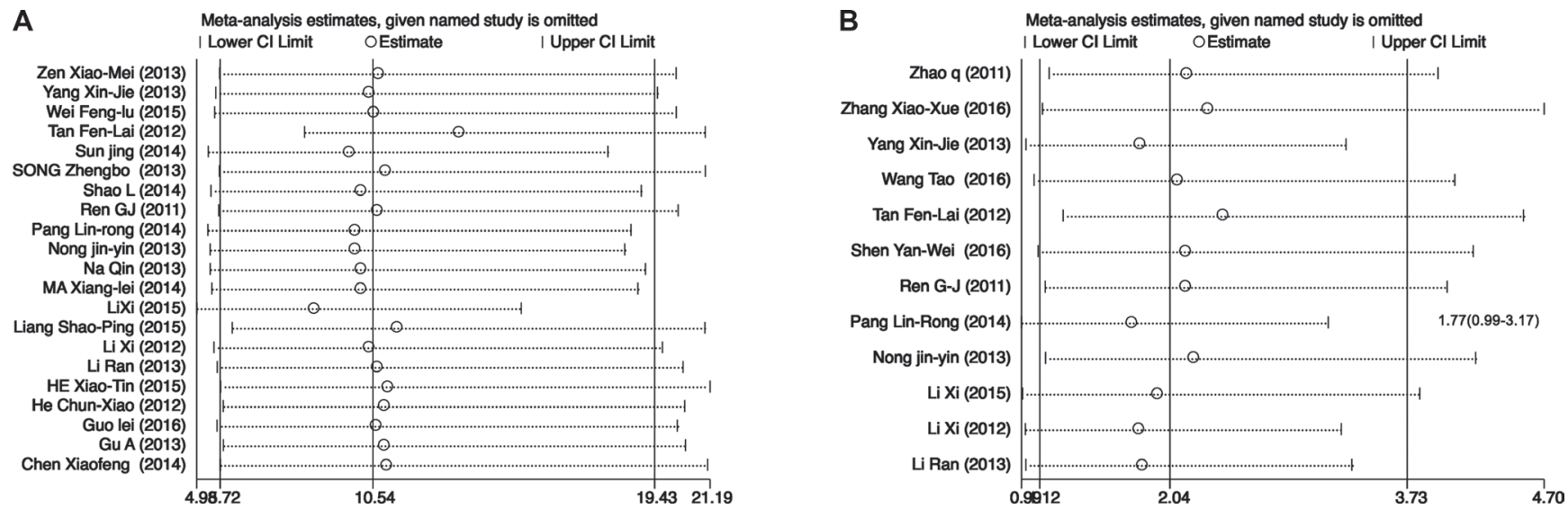

Figure 7: The sensitivity analysis of pooling ORs of DCRs in EGFR mutant patients vs. EGFR wild type patients (A) and ORRs in EGFR 19Del and EGFR L858R patients (B). CI: confidence interval. 
heterogeneity when we pooling the ORs of ORRs in EGFR wild type and mutant patients, the ORs of DCRs in EGFR 19Del and L858R patients. Meta-analysis results showed significant between-study heterogeneity in pooling analysis about DCRs in EGFR wild type and mutant patients and the ORRs in EGFR 19Del and L858R patients. Therefore, Mantel-Haenszel random-effects model was used for analysis. In order to figure out the source of heterogeneity in pooling ORs of DCRs in EGFR wild type and mutant patients, we conducted subgroup analysis by NOS. After the subgroup analysis by NOS, there was no heterogeneity in NOS 6 and 5 groups, but there was heterogeneity in NOS 7 group (Figure 3C). In order to draw more cautious conclusion on EGFR status on icotinib efficacy in NSCLC patients, we also performed the sensitivity analysis. We found that excluded studies did not influence the overall effective size in DCRs analysis of EGFR mutant patients vs. EGFR wild type patients (Figure 7A). For heterogeneity of the pooling ORRs in EGFR 19Del and L858R patients, sensitivity analysis showed one publication (Pang LinRong, 2014) [21] could influence the overall effective size in ORRs analysis of EGFR 19Del and EGFR L858R patients(Figure 7B). After we excluded this publication [21], there was no heterogeneity for pooling ORs analysis for ORRs in EGFR 19Del and EGFR L858R patients $\left(\mathrm{I}^{2}=43.8 \%, P=0.058\right)$ (Figure $\left.3 \mathrm{~B}\right)$. We also carried the publication bias analysis by funnel plots, Egger's test and Begg's test. The results showed there was publication bias for pooling ORs analysis for DCRs in the EGFR wild type patients and $E G F R$ mutant patients. Negative results of studies may not be published. Other three groups of pooling ORs analysis showed no publication bias. The inconsistency of these studies may be due to source of patients, disease condition, publication qualities or other clinical issues. Further large sample multi-center studies and well-designed research are needed.

There were some limitations in this meta-analysis. First, the studies enrolled in our meta-analysis were limited. Slight publication bias may exist because the research having negative results may have not been published online. Second, most studies included in our meta-analysis were retrospective and cohort studies, which may have selection bias and blinding bias. The relative small subjects in studies also influence the meta-analysis results.

In conclusion, our meta-analysis suggests that compared with EGFR wild type patients, EGFR mutant patients have better ORRs and DCRs after icotinib treatment; EGFR 19Del patients have better ORRs after icotinib treatment than EGFR L858R patients. EGFR mutation status is a useful biomarker for evaluation of icotinib efficacy in NSCLC patients. Because of limited numbers of studies and small sample sizes included as well as the heterogeneity in our meta-analysis, more randomized and large-scale clinical trials are necessary to confirm our meta-analysis results.

\section{MATERIALS AND METHODS}

\section{Study review and selection}

We reviewed the databases including PubMed, EMBASE, Web of Science, Wanfang and CNKI to 14 Oct. 2016. The searching strategy was "icotinib" or "conmana" and "cancer or carcinoma or tumor". Dr. Jian Qu and Dr. Ya-Nan Wang reviewed all relevant articles to identify potential eligible studies.

\section{Inclusion and exclusion criteria}

The inclusion criteria were including as follows: clinical study on NSCLC patients harboring EGFR status ( $E G F R$ wild type is defined as no mutation for 19Del and L858R; EGFR mutation is defined as harboring EGFR $19 \mathrm{Del}$ or L858R mutation) using icotinib treatment; at least have the one clinical indicator (ORR and DCR). A study was excluded if it was not relevant to cancer and clinical patients, EGFR status, or had no clinical indicators; involved just in animals or cells; or was a review, or abstract having no data. Different opinions on selections were solved by all author's discussion.

\section{Data collection, quality assessment and assessment of risk of bias.}

Therapeutic efficacy was evaluated by response evaluation criteria in solid tumors (RECISTC) including complete response (CR), partial response (PR), stable disease (SD) and progressive disease (PD). Clinical outcome indicators include objective response rate (ORR) and disease control rate (DCR). The data on authors' names, sex, smoking status. Two investigators (Dr. Qu Jian and Dr. Ya-Nan Wang) independently extracted $E G F R$ mutation type, numbers and clinical outcomes. The Newcastle-Ottawa Scale (NOS) method was used to evaluate the quality of selected studies. According to the Cochrane Handbook version 5.1.0 [36], the risk of bias was assessed including method of random sequence generation (selection bias), allocation concealment (selection bias), blinding (performance bias and detection bias), incomplete outcome data (detection bias), and selective reporting (detection bias). We evaluated methodological quality as low, high, or unclear risk of bias.

\section{Statistical analysis}

We used STATA version 12 (Stata Corp, College Station, TX, USA) to carry out the meta-analysis. Heterogeneity was assessed by the Cochrane's $Q$-statistic test and $I^{2}$ test. We used a Mantel-Haenszel random effects model in the analysis if $P<0.05$ and $I^{2}>50 \%$, otherwise, a Mantel-Haenszel fixed effects model was chosen [37]. Count data calculate the relative risk (RR) 
or odds ratio (OR) and expressed as 95\% confidence interval (CI). Publication bias was analyzed by funnel plot, Egger's test and Begg's test. Sensitivity analysis was performed to assess the influence of a single study on the overall effective size. Tests were two-sided and statistical significance was accepted at $P<0.05$.

\section{ACKNOWLEDGMENTS AND FUNDING}

This work was supported by grants of the National Natural Scientific foundation of China (No. 81503166, 81603208) and the Youth Foundation of Xiangya Hospital in Central South University (2014Q08).

\section{CONFLICTS OF INTEREST}

The authors declare no conflicts of interest.

\section{REFERENCES}

1. Jemal A, Siegel R, Xu J, Ward E. Cancer statistics, 2010. CA Cancer J Clin. 2010; 60:277-300.

2. Socinski MA, Crowell R, Hensing TE, Langer CJ, Lilenbaum R, Sandler AB, Morris D. American College of Chest P. Treatment of non-small cell lung cancer, stage IV: ACCP evidence-based clinical practice guidelines (2nd edition). Chest. 2007; 132:277S-289S.

3. Herbst RS, Heymach JV, Lippman SM. Lung cancer. The N Engl J Med. 2008; 359:1367-1380.

4. Zhou C, Wu YL, Chen G, Feng J, Liu XQ, Wang C, Zhang S, Wang J, Zhou S, Ren S, Lu S, Zhang L, Hu C, et al. Erlotinib versus chemotherapy as first-line treatment for patients with advanced EGFR mutation-positive non-smallcell lung cancer (OPTIMAL, CTONG-0802): a multicentre, open-label, randomised, phase 3 study. Lancet Oncol. 2011; 12:735-742.

5. Mitsudomi T, Morita S, Yatabe Y, Negoro S, Okamoto I, Tsurutani J, Seto T, Satouchi M, Tada H, Hirashima T, Asami K, Katakami N, Takada M, et al. Gefitinib versus cisplatin plus docetaxel in patients with non-small-cell lung cancer harbouring mutations of the epidermal growth factor receptor (WJTOG3405): an open label, randomised phase 3 trial. Lancet Oncol. 2010; 11:121-128.

6. Mok TS, Wu YL, Thongprasert S, Yang CH, Chu DT, Saijo N, Sunpaweravong P, Han B, Margono B, Ichinose Y, Nishiwaki Y, Ohe Y, Yang JJ, et al. Gefitinib or carboplatinpaclitaxel in pulmonary adenocarcinoma. N Engl J Med. 2009; 361:947-957.

7. Xue ZX, Wen WX, Zhuang Y, Hua ZJ, Xia YN. Comparison of the efficacy of icotinib in patients with non-small-cell lung cancer according to the type of epidermal growth factor receptor mutation. Mol Clin Oncol. 2016; 5:265-268.

8. Shi Y, Zhang L, Liu X, Zhou C, Zhang L, Zhang S, Wang D, Li Q, Qin S, Hu C, Zhang Y, Chen J, Cheng Y, et al. Icotinib versus gefitinib in previously treated advanced non-small-cell lung cancer (ICOGEN): a randomised, double-blind phase 3 non-inferiority trial. Lancet Oncol. 2013; 14:953-961.

9. Wang X, Niu H, Fan Q, Lu P, Ma C, Liu W, Liu Y, Li W, $\mathrm{Hu}$ S, Ling Y, Guo L, Ying J, Huang J. Predictive value of EGFR overexpression and gene amplification on icotinib efficacy in patients with advanced esophageal squamous cell carcinoma. Oncotarget. 2016; 7:24744-24751. doi: 10.18632/oncotarget.8271.

10. Chen D, Song Z, Cheng G. Clinical efficacy of firstgeneration EGFR-TKIs in patients with advanced nonsmall-cell lung cancer harboring EGFR exon 20 mutations. Onco Targets Ther. 2016; 9:4181-4186.

11. Jiang X, Wang W, Zhang Y. [Clinical Analysis of Icotinib on Beneficiary of Advanced Non-small Cell Lung Cancer with EGFR Common Mutation]. Chin J Lung Cancer. 2016; 19:200-206.

12. Wang $\mathrm{T}$, Wang $\mathrm{RM}$, Dong $\mathrm{ZH}$, Liang NC, Chang $\mathrm{P}$. Complete remission through icotinib treatment in Nonsmall cell lung cancer epidermal growth factor receptor mutation patient with brain metastasis: A case report. Open Med. 2016; 11:11-15.

13. Wang W, Jiang X, Song Z, Zhang Y. Patients harboring EGFR mutation after primary resistance to crizotinib and response to EGFR-tyrosine kinase inhibitor. Onco Targets Ther. 2016; 9:211-215.

14. Shi Y, Sun Y, Ding C, Wang Z, Wang C, Wang Z, Bai C, Bai C, Feng J, Liu X, Li F, Yang Y, Shu Y, et al. China Experts Consensus on Icotinib for Non-small Cell Lung Cancer Treatment (2015 version). [Article in Chinese] Chin J Lung Cancer. 2015; 18:397-400.

15. Chen LG, Ye H, Zhu Y, Gao MJ, Yang JZ, Lin YX, Chen DW, Sun LR, Hu GX, Xiong JH. Sex Differences in the Pharmacokinetics of Icotinib, a Novel EGFR-TKI, in Rats. Lat Am J Pharm. 2016; 35:675-679.

16. Qin N, Yang X, Zhang Q, Li X, Zhang H, Lv J, Wu Y, Wang J, Zhang S. Efficacy of Icotinib treatment in patients with stage IIIb/IV non-small cell lung cancer. Thoracic cancer. 2014; 5:243-249.

17. Shen YW, Zhang XM, Li ST, Lv M, Yang J, Wang F, Chen ZL, Wang BY, Li P, Chen L, Yang J. Efficacy and safety of icotinib as first-line therapy in patients with advanced non-small-cell lung cancer. Onco Targets Ther. 2016; 9:929-935.

18. Li X, Qin N, Wang J, Yang X, Zhang X, Lv J, Wu Y, Zhang H, Nong J, Zhang Q, Zhang S. Clinical Observation of Icotinib Hydrochloride for Advanced Non-small Cell Lung Cancer Patients with EGFR Status Identified. [Article in Chinese] Chin J Lung Cancer. 2015; 18:734-739.

19. Yang S, Hu X, Li J, Wang Z, Wang Y, Hao X, Wang H, Xu J, Wang B, Lin L, Zhang X, Zhou S, Liu P, et al. 3058 Patient characteristics and treatment outcome of advanced nonsquamous non-small cell lung cancer with over 6-month disease control from icotinib. Eur J Cancer. 2015; 51:S615.

20. Zhao Q, Shentu J, Xu N, Zhou J, Yang G, Yao Y, Tan F, Liu D, Wang Y, Zhou J. Phase I study of icotinib 
hydrochloride (BPI-2009H), an oral EGFR tyrosine kinase inhibitor, in patients with advanced NSCLC and other solid tumors. Lung cancer. 2011; 73:195-202.

21. Pang LRC J, Huang J, Xu CH, Li H, Zhen HY. Icotinib hydrochloride monotherapy for patients with advanced nonsmall cell lung cancer. Zhejiang Med J. 2015; 20:1668-1680.

22. Nong J, Qin N, Wang J, Yang X, Zhang H, Wu Y, Lv J, Zhang Q, Zhang S. Clinical effects for patients with recurrent advanced non-small cell lung cancer treated with icotinib hydrochloride. [Article in Chinese] Chin J Lung Cancer. 2013; 16:240-245.

23. Yarden Y, Shilo BZ. SnapShot: EGFR signaling pathway. Cell. 2007; 131:1018.

24. Kim ES, Hirsh V, Mok T, Socinski MA, Gervais R, Wu YL, Li LY, Watkins CL, Sellers MV, Lowe ES, Sun Y, Liao ML, Osterlind K, et al. Gefitinib versus docetaxel in previously treated non-small-cell lung cancer (INTEREST): a randomised phase III trial. Lancet. 2008; 372:1809-1818.

25. Ciuleanu T, Stelmakh L, Cicenas S, Miliauskas S, Grigorescu AC, Hillenbach C, Johannsdottir HK, Klughammer B, Gonzalez EE. Efficacy and safety of erlotinib versus chemotherapy in second-line treatment of patients with advanced, non-small-cell lung cancer with poor prognosis (TITAN): a randomised multicentre, open-label, phase 3 study. Lancet Oncol. 2012; 13:300-308.

26. Shepherd FA, Rodrigues Pereira J, Ciuleanu T, Tan EH, Hirsh V, Thongprasert S, Campos D, Maoleekoonpiroj S, Smylie M, Martins R, van Kooten M, Dediu M, Findlay B, et al. Erlotinib in previously treated non-small-cell lung cancer. N Engl J Med. 2005; 353:123-132.

27. Hirsch FR, Varella-Garcia M, Bunn PA, Jr., Franklin WA, Dziadziuszko R, Thatcher N, Chang A, Parikh P, Pereira JR, Ciuleanu T, von Pawel J, Watkins C, et al. Molecular predictors of outcome with gefitinib in a phase III placebocontrolled study in advanced non-small-cell lung cancer. J Clin Oncol. 2006; 24:5034-5042.

28. Rosell R, Carcereny E, Gervais R, Vergnenegre A, Massuti B, Felip E, Palmero R, Garcia-Gomez R, Pallares C, Sanchez JM, Porta R, Cobo M, Garrido P, et al. Erlotinib versus standard chemotherapy as first-line treatment for European patients with advanced EGFR mutation-positive non-small-cell lung cancer (EURTAC): a multicentre, open-label, randomised phase 3 trial. Lancet Oncol. 2012; 13:239-246.

29. Shi Y, Au JS, Thongprasert S, Srinivasan S, Tsai CM, Khoa MT, Heeroma K, Itoh Y, Cornelio G, Yang PC. A prospective, molecular epidemiology study of EGFR mutations in Asian patients with advanced non-small-cell lung cancer of adenocarcinoma histology (PIONEER). J Thorac Oncol. 2014; 9:154-162.

30. Tanaka T, Matsuoka M, Sutani A, Gemma A, Maemondo M, Inoue A, Okinaga S, Nagashima M, Oizumi S, Uematsu K, Nagai Y, Moriyama G, Miyazawa H, et al. Frequency of and variables associated with the EGFR mutation and its subtypes. Int J Cancer. 2010; 126:651-655.
31. Yao S, Qian K, Wang R, Li Y, Zhang Y. Comparison of the Efficacy and Safety of Icotinib with Standard Second-line Chemotherapy in Previously Treated Advanced Non-small Cell Lung Cancer. [Article in Chinese] Chin J Lung Cancer. 2015; 18:369-373.

32. Wang T, Liu Y, Zhou B, Wang Z, Liang N, Zhang Y, Dong Z, Li J. Effects of icotinib on early-stage non-smallcell lung cancer as neoadjuvant treatment with different epidermal growth factor receptor phenotypes. Onco Targets Ther. 2016; 9:1735-1741.

33. Chen X, Zhu Q, Liu Y, Liu P, Yin Y, Guo R, Lu K, Gu Y, Liu L, Wang J, Wang Z, Roe OD, Shu Y, Zhu L. Icotinib is an active treatment of non-small-cell lung cancer: a retrospective study. PloS one. 2014; 9:e95897.

34. Li X, Yang XJ, Sun YF, Qin N, Lu JL, Wu YH, Zhang H, Zhang Q, Zhang SC. Clinical observation of icotinib hydrochloride for patients with advanced non-small cell lung cancer. [Article in Chinese] Chin J Lung Cancer. 2012; 34:627-631.

35. Tan F, Shen X, Wang D, Xie G, Zhang X, Ding L, Hu Y, He W, Wang Y, Wang Y. Icotinib (BPI-2009H), a novel EGFR tyrosine kinase inhibitor, displays potent efficacy in preclinical studies. Lung cancer. 2012; 76:177-182.

36. S.Green. JPTH. CochranceHandbook for Systematic Reviews of Interventions Version 5.1.0 [EB/OL]. The Cochrane Collaboration. 2011; http://www.cochrane-handbook.org.

37. Ford AC, Forman D, Hunt RH, Yuan Y, Moayyedi P. Helicobacter pylori eradication therapy to prevent gastric cancer in healthy asymptomatic infected individuals: systematic review and meta-analysis of randomised controlled trials. BMJ. 2014; 348:g3174.

38. Zeng XMH, Li M, ZX. Pu, D. Efficacy of icotinib for advanced lung adenocarcinoma. Sichuan J Cancer Control. 2013; 26:12-15.

39. Yang X, Zhang H, Qin N, Li X, Nong J, Lv J, Wu Y, Zhang Q, Zhang S. [Clinical observation of icotinib hydrochloride in first-line therapy for pulmonary adenocarcinoma. [Article in Chinese] Chin J Lung Cancer. 2013; 16:364-368.

40. Wei FLC, Wang JL, Zou QS, Z.T. Effect of icotinib hydrochloride targeted therapy on non small cell lung cancer. J Haerbin Med Univ. 2015; 49:364-366.

41. Sun JC, WuH XF, Gu YH, Shu YQ, Liu LK. Clinical study of icotinib in treatment of advanced non-small cell lung cancer. China J Mod Dr. 2014; 52:116-119.

42. Song Z, Yu X, Cai J, Shao L, Lin B, He C, Zhang B, Zhang Y. Efficacy of icotinib for advanced non-small cell lung cancer patients with EGFR status identified. [Article in Chinese] Chin J Lung Cancer. 2013; 16:138-143.

43. Shao L, Zhang B, He C, Lin B, Song Z, Lou G, Yu X, Zhang Y. Efficacy and safety of icotinib in Chinese patients with advanced non-small cell lung cancer after failure of chemotherapy. Chin Med J. 2014; 127:266-271. 
44. Ren GJ, Zhao YY, Zhu YJ, Xiao Y, Xu JS, Shan B, Zhang L. Tumor gene mutations and messenger RNA expression: correlation with clinical response to icotinib hydrochloride in non-small cell lung cancer. Chin Med J. 2011; 124:19-25.

45. Ma XLS, MQ, Kou YY, Tang YQ. Efficacy and safety of icotinib in patients with advanced non-small cell lung adenocarcinoma. Anhui Med Pharm J. 2014; 18:2174-2177.

46. Liang SPW, HQ. Clinical observation of icotinib hydrochloride in the treatment of advanced non-small cell lung cancer. J Tianjin Med Univ. 2015; 21:51-55.

47. LI RP, ZY, Ren SS, Liang H, Sun JD. Observation on efficacy of icotinib hydrochloride for treating advanced non-small cell lung cancer. J Mod Med Health. 2013; 29:2255-2259.
48. HE XTL, CY, Lu PH, Hang ZQ. Efficacy of Icotinib in Treatment of Advanced Non-small Cell Lung Cancer and Prognosis Evaluation. Chin Gen Pract. 2015; 18:998-1101.

49. He CXZ, BB, Zheng L, Song ZB, Shao L, Lin BC, Lou GY, Zhao J, Zhang YP. Efficacy of icotinib hydrochloride in the treatment of 31 patients with advanced squamous cell lung carcinoma. Chin J New Drugs. 2013; 22:1430-1434.

50. Guo LL, AJ, Wang JW, Tao HT, Hu YA. retrospective study of icotinib for the treatment of advanced non-small cell lung adenocarcinoma. Chin J Drug Appl Monit. 2015; 12:69-72.

51. Gu A, Shi C, Xiong L, Chu T, Pei J, Han B. Efficacy and safety evaluation of icotinib in patients with advanced nonsmall cell lung cancer. Chin J Cancer Res. 2013; 25:90-94. 